\title{
Malignant Reproductive System Neoplasm
}

National Cancer Institute

\section{Source}

National Cancer Institute. Malignant Reproductive System Neoplasm. NCI Thesaurus.

Code C36076.

A primary or metastatic malignant neoplasm that affects the male or female reproductive system. Representative examples include prostate carcinoma, penile carcinoma, testicular seminoma, testicular embryonal carcinoma, endometrial carcinoma, cervical carcinoma, ovarian carcinoma, and uterine corpus leiomyosarcoma. 